\title{
Experimental and numerical study of error fields in the CNT stellarator
}

\author{
K C Hammond ${ }^{1}$, A Anichowski ${ }^{1}, \mathbf{P}$ W Brenner ${ }^{1}$, T S

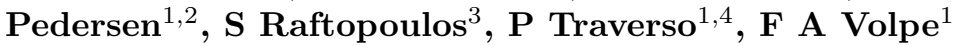 \\ ${ }^{1}$ Dept. of Applied Physics and Applied Mathematics, Columbia University, New \\ York, NY 10027, USA \\ 2 [Present institution: Max Planck Institute for Plasma Physics, 17491 \\ Greifswald, Germany] \\ ${ }^{3}$ Princeton Plasma Physics Laboratory, Princeton, NJ 08543, USA \\ 4 [Present institution: Dept. of Physics, Auburn University, Auburn, AL 36849, \\ USA]
}

\begin{abstract}
Sources of error fields were indirectly inferred in a stellarator by reconciling computed and numerical flux surfaces. Sources considered so far include the displacements and tilts of the four circular coils featured in the simple CNT stellarator. The flux surfaces were measured by means of an electron beam and fluorescent rod, and were computed by means of a Biot-Savart fieldline tracing code. If the ideal coil locations and orientations are used in the computation, agreement with measurements is poor. Discrepancies are ascribed to errors in the positioning and orientation of the in-vessel interlocked coils. To that end, an iterative numerical method was developed. A Newton-Raphson algorithm searches for the coils' displacements and tilts that minimize the discrepancy between the measured and computed flux surfaces. This method was verified by misplacing and tilting the coils in a numerical model of CNT, calculating the flux surfaces that they generated, and testing the algorithm's ability to deduce the coils' displacements and tilts. Subsequently, the numerical method was applied to the experimental data, arriving at a set of coil displacements whose resulting field errors exhibited significantly improved agreement with the experimental results.
\end{abstract}




\section{Introduction}

Error fields (EFs) have been and are the subject of intense study in tokamaks $[1,2]$ where relative errors as small as $10^{-4}$ or even $10^{-5}$ are known to affect stability, cause disruptions, and degrade confinement and plasma rotation. Error fields are obviously also very important in modern transport-optimized stellarators [3, 4], whose performances rely on carefully optimized 3D magnetic fields.

The specially shaped coils that generate such fields are numerically optimized and, typically, are built and positioned with very high precision. In other words, errors are minimized at the construction stage. Vacuum fields are then experimentally characterized by a standard technique involving an electron beam and a fluorescent rod $[5,6,7,8]$. Incidentally, it should be noted that, since this technique requires flux surfaces to exist in a vacuum, it is not applicable to devices such as tokamaks that require plasma current to generate flux surfaces. The measured surfaces are usually confirmed to be in good agreement with the desired, optimal configuration. However, if not in agreement, a comparison of computed and measured flux surfaces can shed light on possible sources of errors. At that point one can either (1) correct the error "at the source" (reposition one or more coils) [9] or (2) apply EF corrections by means of a separate set of dedicated coils. This latter approach is quite common in tokamaks $[1,2,10]$. As for stellarators, EF corrections were applied in LHD by means of Resonant Magnetic Perturbation (RMP) coils, also deployed in other MHD studies [11]. W7-AS used "special" and "control" coils to vary the toroidal mirror term and boundary island geometry and study their effect on plasma properties [12], and similar uses are envisioned for the "trim coils" in W7-X [13]. Earlier possibilities for EF correction in W7-X were discussed in Ref. [14].

Stellarators are considered complicated to build. It has been suggested that their attractiveness as reactors could increase if their construction is simplified, or construction tolerance relaxed, without significantly degrading the plasma properties [15]. At this point it is highly hypothetical, but a possible route to simplification could consist in: i) slightly relaxing the tolerance and ii) correcting the errors a posteriori, either by approach (1) or (2) listed above. In either approach, it is useful to experimentally quantify the displacements, tilts and, possibly, deformations of the actual coils, compared with design values. This is useful anyway, even if stricter tolerance is adopted, to confirm that the construction imperfections are indeed smaller.

As a first step in exploring such route, we have complemented the well-established experimental technique mentioned above with metrology measurements and with a numerical method that "inverts" the flux surface errors into error sources such as imperfections in coils' positions and tilts.

The CNT stellarator, constructed in 2004 [16], is notable for its simple coil configuration and for having the lowest aspect ratio of any stellarator ever built [8]. The magnetic field is generated by a system of four planar, circular coils: two interlocked (IL) coils and two poloidal field (PF) coils, the latter of which form a Helmholtz pair (Fig. 1). This configuration, as designed, generates a set of toroidal nested closed flux surfaces with two field-periods. The radial profile of rotational transform $t$, as well as the general shape of the flux surfaces, can be controlled by varying two main parameters: the tilt angle between the IL coils and the ratio of the current in the IL coils to the current in the $\mathrm{PF}$ coils, $I_{I L} / I_{P F}$. CNT was constructed to permit three different IL coil tilt angles $\theta_{\text {tilt }}: 64^{\circ}, 78^{\circ}$, and $88^{\circ}$. These angles were chosen for their distinct $t$ profiles as well as for their resiliency to EFs [16].

Error field resiliency was an especially important consideration for CNT's coil configurations due to the permissive tolerances used in the construction of the coils and the vacuum vessel. Whereas present-day stellarators are typically built to tolerances on the order of $10^{-3}$ to $10^{-4}[17,18]$, CNT's tolerances were of order $10^{-2}$ to $10^{-3}$ to minimize the cost and complexity of construction.

From 2005-2010, the interlocked coils were kept in the $\theta_{\text {tilt }}=64^{\circ}$ configuration. The flux surfaces for this configuration were measured experimentally and found to agree very well with numerical predictions [8]. More recently, CNT's coils were switched to the $\theta_{\text {tilt }}=78^{\circ}$ configuration. This configuration is predicted to have less magnetic shear than the previous one. As a result, EFs that resonate with rational surfaces within the $t$ profile are expected to have more significant effects on the magnetic geometry, leading to larger islands and equilibrium deformation.

A detailed understanding of the magnetic geometry in CNT is a central objective of the CNT research program. It will allow for more precise alignment of 


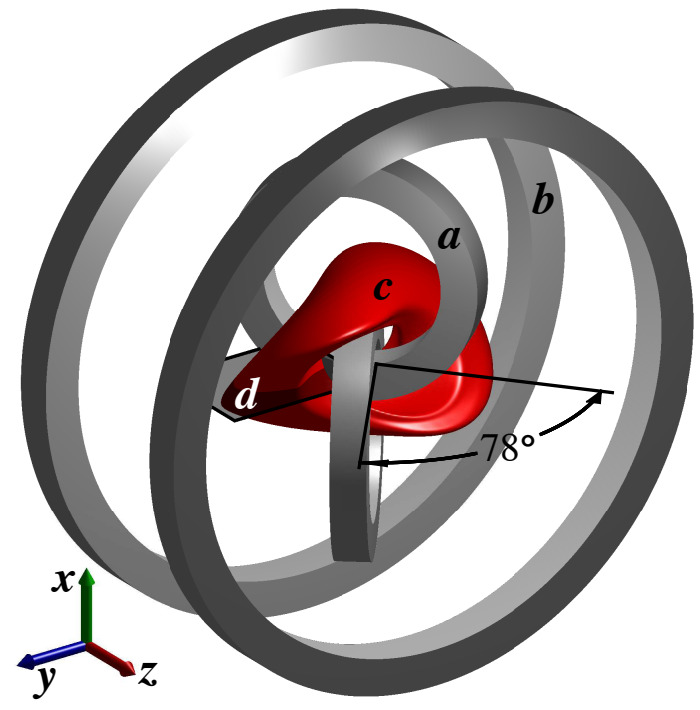

Figure 1. Schematic of the CNT coil configuration. (a) interlocked (IL) coils, (b) poloidal field (PF) coils, (c) the last closed flux surface, (d) plane at $\phi=90^{\circ}$ at which Poincaré crosssections are measured as described in Section 2.

diagnostics and improve the accuracy of equilibrium modeling and reconstruction. It will also assist in the design of equipment whose shaping and placement are heavily dependent on the field, such as island divertors.

In this paper we present the first detailed measurements of the flux surface geometry of the $\theta_{\text {tilt }}=78^{\circ}$ configuration, which exhibits noticeable disagreements with predictions (Sec. 2). We then describe efforts to diagnose the sources of the EFs under the assumption that the main sources of error are displacements of the coils from their design positions. These efforts include (1) photogrammetric measurements of the positions of the PF coils (Sec. 3), (2) studies of the effects of different classes of displacements on the rotational transform $t$ (Sec. 4), and (3) design of an optimization algorithm to find the most likely displacements of the IL coils that lead to the observed flux surface geometry (Sec. 5). We then discuss the potential broader applicability of the optimization algorithm to more complex coil configurations (Sec. 6) and briefly describe future work (Sec. 7).

\section{Flux Surface Measurments}

\subsection{Experimental setup}

The flux surface geometry in CNT is measured with a standard technique involving an electron beam and a fluorescent rod $[8,7]$. Electrons are emitted from an electron gun at energies of roughly $80 \mathrm{eV}$. The electrons travel along a field line until they strike an obstacle, either a part of the vessel (if an open field line) or an aluminum rod that extends into the confining region. The aluminum rod is coated with $\mathrm{ZnO}: \mathrm{Zn}$ fluorescent powder that emits blue-green light (505 nm) when struck by electrons. In CNT, two fluorescent rods are positioned at the mid-plane of the vacuum vessel, which coincides with the toroidal cross-section at $\phi=90^{\circ}$ (Fig. 1). The rods can be rotated across this surface with an external actuation mechanism described in Ref. [8]. Background pressures are maintained at the base pressure of the CNT vacuum vessel $\left(<10^{-8}\right.$ Torr $)$ to minimize collisions with neutral atoms and molecules.

If a long-exposure photograph is taken of the fluorescent rods as they are scanned across the $\phi=90^{\circ}$ plane during the emission of an electron beam, the resulting image will show a cross-section of the flux surface on which the beam was emitted. Certain parts of the cross-section may not appear in the image due to shadowing by the electron gun or the limited extent of the fluorescent rods. For the measurements described in this paper, a digital camera was positioned outside the vacuum vessel to face the plane of the cross-section through a fused-silica viewport. Images were acquired using ten seconds of exposure time. In addition to the fluorescent glow from the rods, the images contained some regions of stray light. This was due to blackbody emission from the electron gun filament reflecting off components in the vacuum vessel. Regions of stray light were eliminated manually from each image before processing.

The relative positions of each pixel were determined based on the length of the rod in the image. The absolute positions of each pixel were then determined by comparing the position of the rod to the position of a fixed landmark in the chamber. The lens axis of the camera was confirmed to be within one degree of perpendicular to the $\phi=90^{\circ}$ plane through the use of a leveling tool. This uncertainty could contribute to up to $3 \mathrm{~mm}$ of displacement of the resulting image, as the camera was placed roughly one meter away from the cross-section.

\subsection{Results}

Two examples of experimentally measured Poincaré cross-sections are shown in Fig. 2, overlaid with numerically computed Poincaré data for the corresponding current-ratios. The numerical data, determined using a Biot-Savart field line tracer described in Ref. [19], were generated assuming that the coils were perfectly aligned. Note that the qualitative agreement is poor: the outboard side of the surfaces at $I_{I L} / I_{P F}=3.68$ (Fig. 2a) are flatter and have more vertical elongation than the experimental surfaces. In addition, the experimental data for $I_{I L} / I_{P F}=3.18$ (Fig. $2 \mathrm{~b}$ ) have promi- 

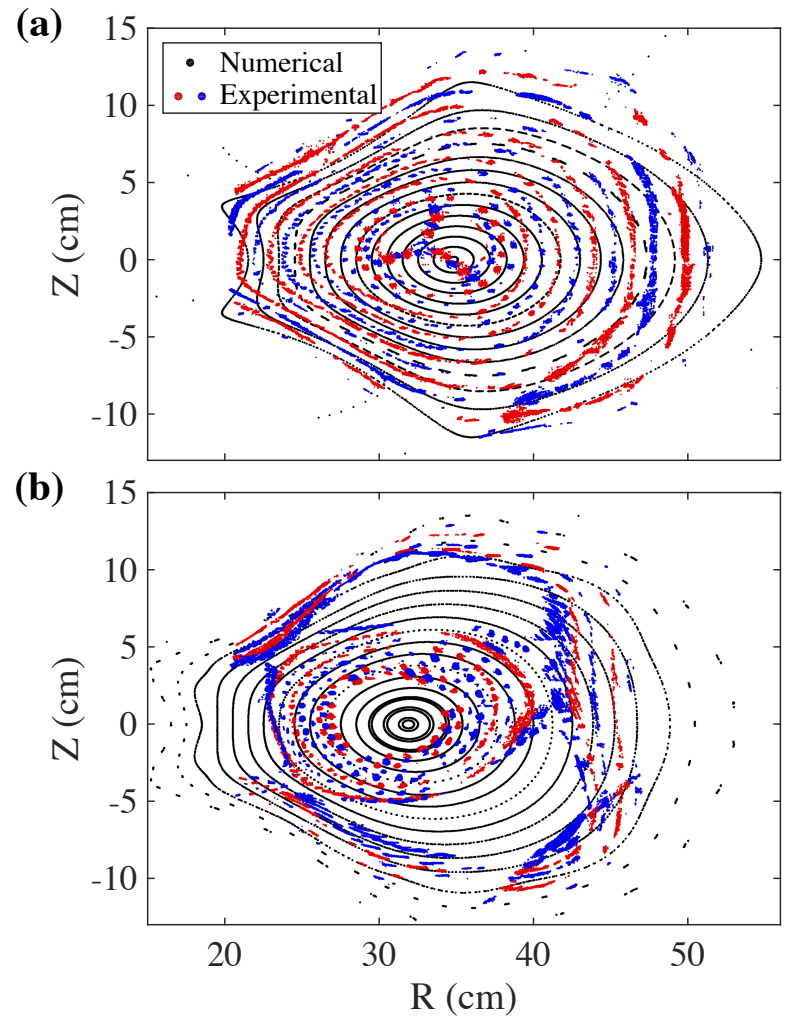

Figure 2. Comparison of numerical Poincaré plots (black dots) with experimental results (adjacent surfaces are shown in alternating red and blue for clarity). Numerical data were determined assuming that the coils were perfectly aligned. (a) $I_{I L} / I_{P F}=3.68$; (b) $I_{I L} / I_{P F}=3.18$

nent islands that are not predicted numerically.

In addition to the geometric disagreements in the Poincaré sections at toroidal angle $\phi=90^{\circ}$, the measured rotational transform $t$ has been observed to differ from numerical predictions. While detailed profiles of $t$ have not been measured in CNT, it is possible to identify low-order rational surfaces through field line visualizations [20]. In this technique, the electron gun is operated while the vacuum vessel is back-filled to a neutral pressure between $10^{-5}$ and $10^{-4}$ Torr. At these pressures, electron-neutral collisions are frequent enough that the path of the electron beam emits a glow that is visible to the naked eye. In the vicinity of a low-order rational surface, the beam can be seen to strike the back side of the electron gun after a finite number of toroidal transits. For example, for $I_{I L} / I_{P F}<3.5$, a region exists in which the electron beam is observed to strike the electron gun after three toroidal transits, indicating the presence of a surface (or island chain) with $t=1 / 3$. On the other hand, numerically calculated $t$ profiles (Fig. 3) only contain $t=1 / 3$ for $I_{I L} / I_{P F}<3.18$. Evidently, an $\mathrm{EF}$ is causing a systematic offset of the rotational transform.

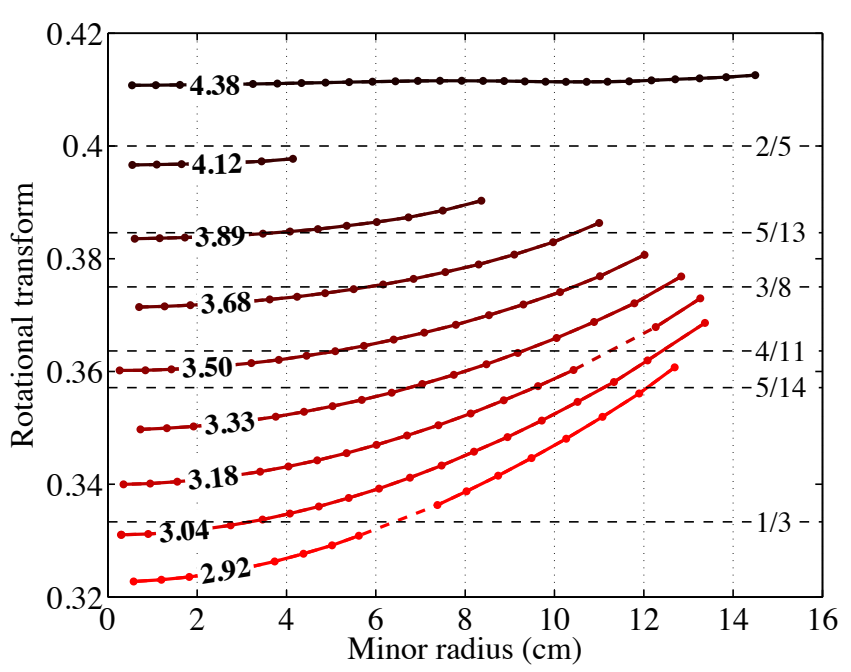

Figure 3. Plots of $t$ profiles for selected current-ratios in CNT in the $78^{\circ}$ IL coil configuration. In the red curves, which are labeled with respective values of $I_{I L} / I_{P F}$, each dot represents a closed flux surface and dashed segments indicate island chains. Some low-order rational numbers are shown as horizontal dashed lines. Note that current-ratios of 3.18 and above are not predicted to contain $t=1 / 3$, contrary to measurements.

The observed discrepancies between experiment and calculations motivated a more detailed study of the possible sources of EFs.

\section{Photogrammetry}

Displacements of the PF coils from their design positions were measured directly using photogrammetry. In this procedure, reflective markers were affixed to all parts of the PF coils as well as the exterior of the vacuum vessel. The vessel and coils were then photographed from several different angles. The photographs were analyzed by V-STARS software (produced by Geodetic Systems, Inc.), which generated a point cloud giving the relative positions of the markers in 3D space. The point cloud was then fitted to the nominal surfaces of the machine as provided by a CAD file. The result was a set of displacement vectors (Fig. 4) for each marker corresponding to the displacement of its respective part of the coil from the design location. Displacement vectors that deviated significantly from those of neighboring markers were viewed as erroneous and were ignored in subsequent analysis.

An attempt was also made to measure the positions of the IL coils using a Romer six-axis measuring arm. However, limited access to the IL coils restricted their measurements to a small region of each coil. The measurements obtained were sufficient to estimate the location of the center of the device to within less than $1 \mathrm{~mm}$ (which was then used as a reference for the PF coil marker offsets), but did 


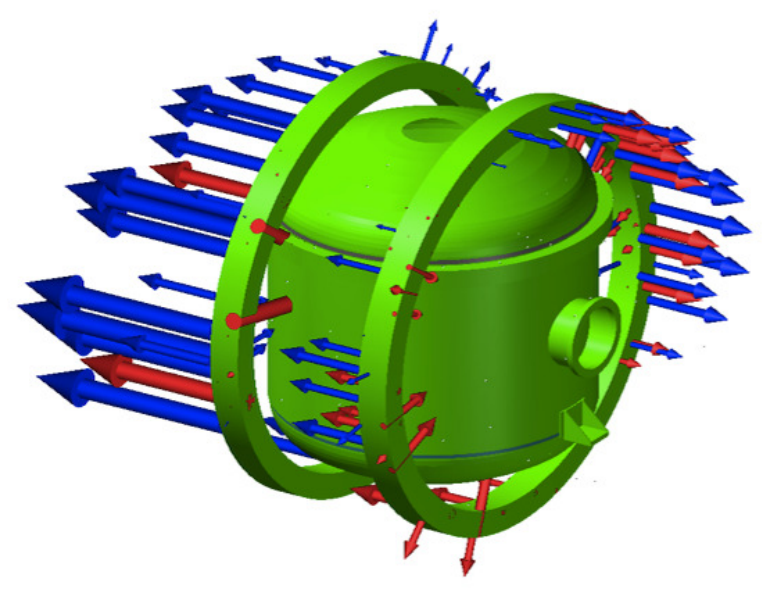

Figure 4. Rendering of the CNT vacuum vessel and PF coils overlaid with arrows showing the displacements of the coils from their design positions. Each arrow corresponds to one photogrammetric marker. Arrow lengths are to scale with one another but are exaggerated relative to the vacuum vessel and coils. The largest arrow shown has a length of $44 \pm 3 \mathrm{~mm}$. Blue (red) arrows represent deviations in the same (opposite) direction of the vector normal to the surface where they originate.

not yield accurate estimates of the misalignments of the IL coils relative to one another. We will note that it is possible to configure the chamber to permit full access for the Romer arm, but this could not be done on the time scale of the arm's availability for the measurements described in this paper and will be an objective for future work.

According to the marker displacements determined by the software, certain regions of the PF coils are separated by as much as $44 \pm 3 \mathrm{~mm}$ from their intended locations, which is well outside of the $10 \mathrm{~mm}$ tolerance specified for construction. It is noteworthy that CNT's coils still produce good flux surfaces despite having displacements of this magnitude.

The observed misalignments of the PF coils are believed to have arisen during a procedure to change the IL coil configuration from $\theta_{\text {tilt }}=64^{\circ}$ to $\theta_{\text {tilt }}=$ $78^{\circ}$. The PF coils must be tilted out of the way to permit access to the IL coils, and they sit on hinged support structures for this purpose. By the end of the procedure, the structures had visibly deformed due to internal stresses arising from the tilting, likely affecting the positions of the PF coils themselves.

To determine the effects of the measured PF coil displacements on the flux surfaces, new field line traces were conducted with the PF coils offset according to the photogrammetry data. For these calculations, the PF coils were still assumed to be circular and planar but were tilted and translated so as to best fit the displacement vectors. According to these fits, the northern $(z>0)$ and southern $(z<0)$ PF coils were (a)
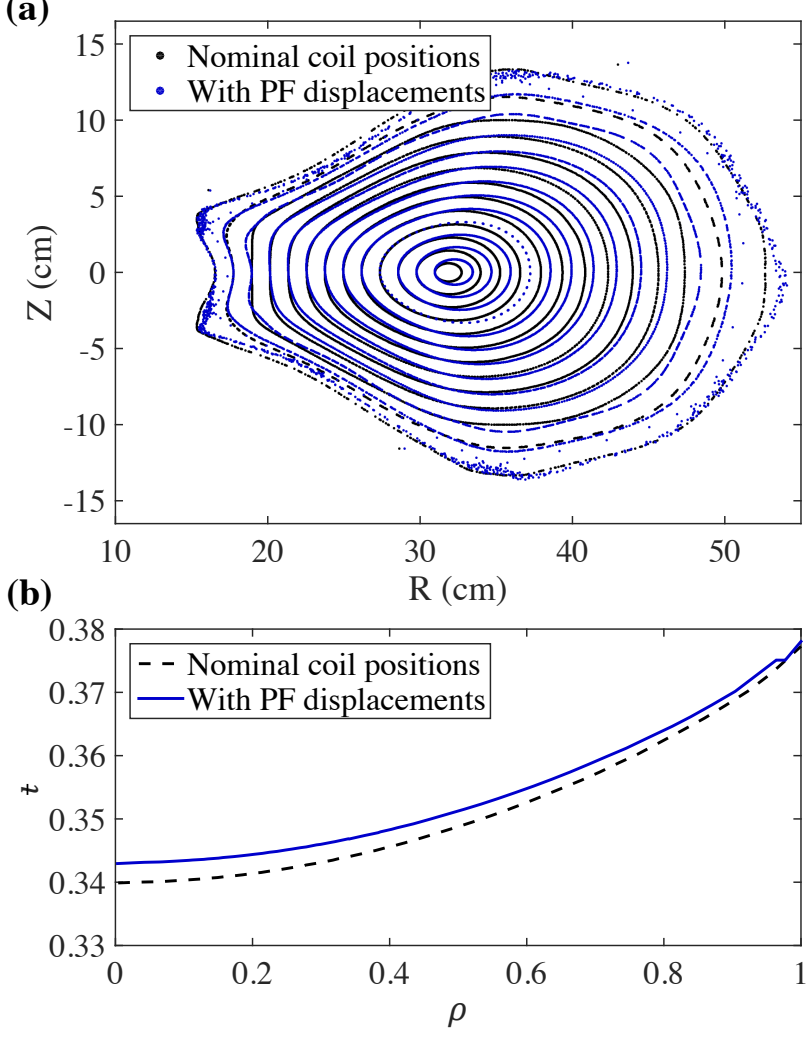

Figure 5. (a) Numerically generated Poincaré plots at the $\phi=90^{\circ}$ cross-section with $I_{I L} / I_{P F}=3.18$ from a configuration incorporating the measured PF coil displacements (blue) and from the design configuration (black). (b) Calculated profiles of $t$ for this configuration.

translated $5 \mathrm{~mm}$ and $22 \mathrm{~mm}$ respectively, and their axes were tilted by $1.0^{\circ}$ and $1.3^{\circ}$ respectively.

A comparison of the numerical Poincaré plots with and without the displacements is shown in Fig. 5 using $I_{I L} / I_{P F}=3.18$. Although the plots are not identical (in particular, the magnetic axis has shifted slightly outboard and field lines initiated at the same locations do not overlap perfectly), the effects of the $\mathrm{PF}$ coil displacements do not appear to be sufficient to explain the observed experimental discrepancies. In particular, the slight increase in the $t$ profile is actually the opposite effect of what would be needed to explain the $t=1 / 3$ island chain seen at an intermediate minor radius in Fig. $2 b$.

Since the PF coil displacements were not enough to explain the observed differences in Poincaré crosssections, additional sources of field error were sought.

\section{Numerical study of $t$ sensitivity}

In the search for additional sources of field error, the most likely candidate was thought to be displacements to the IL coils. Previous work [21] has shown that a 
translational displacement of either of the IL coils has a significantly greater effect on the magnetic geometry than the same displacement applied to either of the PF coils. (For this reason, the tolerance for the IL coils was set at $2 \mathrm{~mm}$, one-fifth of the PF coil tolerance.)

To identify the possible causes of the systematic offset in $t$ discussed in Section 2, a series of numerical field line traces was computed for different classes of IL coil displacements. Field lines were also traced for similar classes of PF coil displacements for comparison. The classes of displacements were sorted into symmetric (equal coil movement in opposite directions) and antisymmetric (equal coil movement in the same direction). For each class of displacement, $t$ profiles were calculated for a series of magnitudes of the displacement. To represent the calculated trends in $t$, Fig. 6 shows derivatives of $t_{\text {axis }}$ (i.e., the limit of $t$ as the minor radius approaches zero) with respect to each type of displacement. Derivatives were estimated by second-order finite differences about the nominal positions of the coils at $I_{I L} / I_{P F}=3.68$.

The displacements plotted in Fig. 6 collectively represent the twenty degrees of freedom that the four coils have for rigid motion (five for each coil; rotation of a coil about its axis is ignored due to symmetry). The translational displacements shown in Fig. 6a have fairly intuitive interpretations. A positive "ILC co$x$ " displacement, for example, involves motion of both the IL coils in the positive $x$ direction as indicated in Fig. 1. An "ILC counter- $x$ " displacement, on the other hand, involves motion in opposite ways in the $x$ direction, with positive displacement indicating the IL coils moving apart and negative displacement indicating the IL coils moving toward one another.

The angular displacements shown in Fig. 6b represent tilts of the coil in two orthogonal directions denoted by $\hat{a}$ and $\hat{b}$. The $\hat{a}$ and $\hat{b}$ vectors for the IL coils are illustrated in Fig. 7. "ILC co-b-tilt" displacement, for example, refers to both IL coils tilting by some angle $\theta$ such that their axes rotate toward their respective $\hat{b}$ unit vectors. A change in ILC co-b-tilt is equivalent to an adjustment of $\theta_{\text {tilt }}$ (nominally $78^{\circ}$ ). "ILC countera-tilt" displacement, as another example, refers to the IL coils tilting such that IL1 tilts its axis in the positive $\hat{a}_{I L 1}$ direction and IL2 tilts its axis in the negative $\hat{a}_{I L 2}$ direction. For both $\mathrm{PF}$ coils, $\hat{a}=\hat{x}$ and $\hat{b}=\hat{y}$.

From Fig. 6, it is evident that the two classes of displacements that have the greatest influence on $t$ are the separation of the IL coils (along the $x$ axis) and the tilt angle between the IL coils. Another lessprominent influence arises from the separation of the $\mathrm{PF}$ coils. This is roughly equivalent to changing the $\mathrm{PF}$ coil current, as it strengthens or weakens the Helmholtz field created by the coils, and illustrates how adjusting the PF current relative to the IL current serves as a (a)

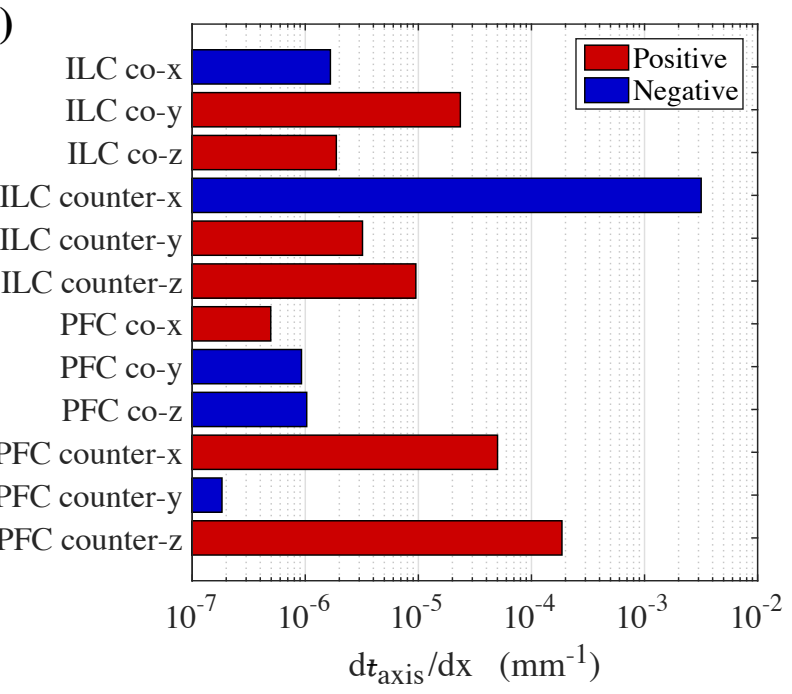

(b)

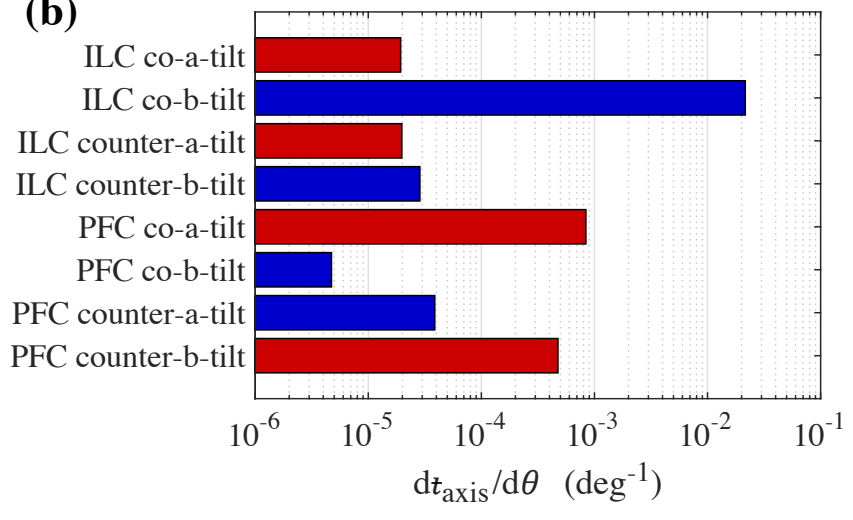

Figure 6. Derivatives of $t_{a x i s}$ with respect to the magnitude of different classes of coil displacements. (a) Translations of the coils in the Cartesian directions illustrated in Fig. 1. (b) Tilts of the coils in the directions of their respective $\hat{a}$ and $\hat{b}$ axes. The $\hat{a}$ and $\hat{b}$ axes for the IL coils are illustrated in Fig. 7; for both PF coils, $\hat{a}=\hat{x}$ and $\hat{b}=\hat{y}$. A more detailed description of each displacement class is given in the text.

fine adjustment to the iota profile.

The relative sensitivity of $t$ to the different classes of coil displacements in Fig. 6 suggests that, if the offset of the $t$ profile is indeed caused by coil displacements, it is very likely that displacements of the IL coils play a significant role. This observation motivated a more detailed study of IL coil displacements to be described in the following section.

\section{Optimization}

\subsection{Optimization procedure}

The optimization method used in this study tweaks the coil positions in small increments until the calculated Poincaré data resulting from the perturbation matches with the experimentally obtained Poincaré 


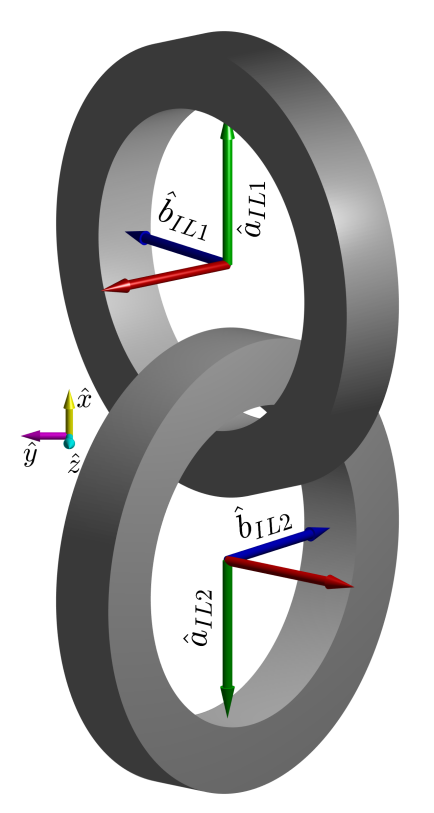

Figure 7. Schematic of the two IL coils along with their respective axes of symmetry (red arrows), $\hat{a}$ vectors (green arrows), and $\hat{b}$ vectors (blue arrows) as described in the text. The $\hat{x}, \hat{y}$, and $\hat{z}$ directions as defined in Fig. 1 are shown in yellow, magenta, and cyan respectively.

cross-sections. The procedure is conceptually similar to methods used in some plasma equilibrium reconstruction codes such as EFIT and V3FIT [22, 23], which determine plasma equilibrium parameters that fit to diagnostic signals. In this procedure, Poincaré cross-sections take the role of the diagnostic signals, whereas coil displacements have the role of the equilibrium parameters.

For the optimizations described in this paper, the observed Poincaré cross-sections are mapped into a vector $\mathbf{X}$ of discrete geometric parameters, which may be thought of equivalently as physics parameters derived from flux surface topology. The definitions of these parameters are provided in Appendix A, and the procedure for determining them from experimental and numerical Poincaré data is given in Appendix B. The coil misalignments were encapsulated in a vector $\mathbf{p}$ of displacement parameters (i.e., engineering parameters). The work in this paper used two variants of $\mathbf{p}$, both of which will be described later in this section.

An optimization begins with target geometric parameters $\mathbf{X}^{*}$ for the desired Poincaré cross-section geometry and an initial guess $\mathbf{p}_{0}$ of coil displacement parameters. The geometric parameters associated with $\mathbf{p}_{0}$ (or any set of dispacement paramameters $\mathbf{p}$ ) are then $\mathbf{X}(\mathbf{p})$. The discrepancy $\mathbf{F}(\mathbf{p})$ between $\mathbf{X}^{*}$ and
$\mathbf{X}(\mathbf{p})$ is defined as

$$
F_{i}(\mathbf{p})=X_{i}(\mathbf{p})-X_{i}^{*}
$$

The optimization algorithm attempts to find $\mathbf{p}^{*}$ such that $\mathbf{F}=0$ using the Newton-Raphson method [24]. Expanding $\mathbf{F}$ about a coil configuration $\mathbf{p}$ that differs from $\mathbf{p}^{*}$ by $\delta \mathbf{p}$, i.e.,

$\mathbf{F}(\mathbf{p}+\delta \mathbf{p})=0=\mathbf{F}(\mathbf{p})+J \delta \mathbf{p}+O\left(\delta \mathbf{p}^{2}\right)$,

the iterative Newton step $\delta \mathbf{p}$ is given by a solution to the equation

$\mathbf{F}(\mathbf{p})=-J \delta \mathbf{p}$.

Here, $J$ is the Jacobian,

$J_{i j}=\frac{\partial F_{i}}{\partial p_{j}}$

In general, $\mathbf{p}$ is not of the same dimension as F. We thus find the optimal $\delta \mathbf{p}$ through linear leastsquares. The Best Linear Unbiased Estimator (BLUE) for $\delta \mathbf{p}$ is that which minimizes $\chi^{2}[25]$ :

$\chi^{2}=\mathbf{F}^{T} C^{-1} \mathbf{F}$

where $C_{i j}=\operatorname{cov}\left(X_{i}^{*}, X_{j}^{*}\right)$ is the covariance matrix for the target parameters $\mathbf{X}^{*}$. This estimator for $\delta \mathbf{p}$ is given by

$\delta \mathbf{p}=-\left(J^{T} C^{-1} J\right)^{-1} J^{T} C^{-1} \mathbf{F}$.

\subsection{Numerical considerations}

5.2.1. The covariance matrix For experimental Poincaré data, the covariance matrix $C$ is estimated by evaluating $\mathbf{X}$ for multiple samples of the pixels obtained from composite flux surface images. For each sample, pixels are selected randomly with replacement (bootstrapping) [26]. The number of pixels available to sample from a particular flux surface in the images used for this analysis ranged from 300 to more than 10,000. Each sample consists of 400 pixels per flux surface.

For the verification studies conducted in Sec. 5.3, the optimizer was programmed to fit the coils to numerically determined Poincaré data (i.e., a manufactured solution). To obtain a covariance matrix for numerical Poincaré data, multiple Poincaré plots are obtained by tracing field lines from randomized initialization points. Each sample consists of a trace of 200 field lines followed for 200 toroidal revolutions. (A certain percentage of the field lines will terminate if they are initialized outside of the last closed flux surface; these are ignored.)

As indicated in Eq. 6, $C$ must be inverted for the analysis. This can introduce significant numerical error if $C$ is ill-conditioned. To mitigate this risk, rather 
than directly inverting $C$, its inverse is approximated through singular value decomposition:

$C=U \Sigma U^{T}$

Here, the orthogonal matrices $U$ to the left and right of $\Sigma$ are the same due to the inherent symmetry of C. $\Sigma$ is a diagonal matrix of the singular values. If $C$ is ill-conditioned, the lowest singular value(s) will be much less than the greatest singular value. As a workaround, a cutoff is imposed such that all singular values of $C$ less than a factor $1 / \kappa_{c o}$ of the greatest singular value are set to zero. The pseudoinverse of this approximation is given by

$C^{-1} \approx U \Sigma^{-1} U^{T}$,

where $\Sigma^{-1}$ is a diagonal matrix in which the $n^{t h}$ nonzero diagonal element is equal to the inverse of the $n^{\text {th }}$ nonzero diagonal element of $\Sigma$. For the work described in this paper, $\kappa_{c o}$ was chosen empirically to be $10^{8}$ for the calculations in this paper as the lowest value for which the Newton direction was not noticeably compromised.

5.2.2. The Jacobian The elements of the Jacobian in this implementation are computed by second-order finite differences:

$J_{i j}=\frac{F_{i}\left(\mathbf{p}+\Delta \mathbf{p}_{j}\right)-F_{i}\left(\mathbf{p}-\Delta \mathbf{p}_{j}\right)}{\left|\Delta \mathbf{p}_{j}\right|}$

Note that, due to its dependence on $\mathbf{X}^{*}$ via $\mathbf{F}$, $J_{i j}$ contains random error and may be correlated with other matrix elements. Because of this, Eq. 6 does not, strictly speaking, give the BLUE for $\delta \mathbf{p}$, since one of the underlying assumptions is that $J$ is non-random. Nevertheless, we have found that this estimator is sufficient in many cases as long as the finite differencing interval $\Delta \mathbf{p}$ is sufficiently large.

5.2.3. Line-search If $\mathbf{F}$ depends nonlinearly on $\mathbf{p}$, there is a risk that the Newton step $\delta \mathbf{p}$ will overshoot a local minimum in $\chi^{2}$. To rectify such occurrences, a line-search algorithm checks the Newton step after each iteration to ensure that the average rate of decrease in $\chi^{2}$ over the interval $\delta \mathbf{p}$ is at least as great as the gradient of $\chi^{2}$ evaluated at the starting point $\mathbf{p}$ of the iteration. If this condition is not met, the algorithm samples $\chi^{2}$ at shorter distances along the direction of the Newton step to identify the local minimum. More details on this algorithm are given in Ref. [24].

\subsection{Verification}

To verify the performance of the algorithm, some tests were conducted in which the code was used to solve for a known coil displacement $\mathbf{p}^{*}$ using target parameters
$X^{*}$ generated from a field-line trace that used $\mathbf{p}^{*}$ as its input.

One early test was meant to determine whether the algorithm could distinguish two classes of displacements that produced qualitatively similar outcomes. One such pair of displacement classes is (1) separation of the coils along the $x$ axis and (2) adjustment of $\theta_{\text {tillt }}$. Incidentally, these two classes were also found to have the greatest impact on rotational transform (Fig. 6), a strong determiner of the cross-section geometry. A p vector was thus defined with just two components:

$\mathbf{p}=\left[\begin{array}{c}\frac{1}{2 c_{x}}\left(\Delta x_{I L 1}-\Delta x_{I L 2}\right) \\ \frac{1}{c_{\theta}} \Delta \theta_{t i l t}\end{array}\right]$

Here, $\Delta x_{I L 1}$ and $\Delta x_{I L 2}$ are the displacements along the machine $x$ axis of the first and second IL coil, respectively, from their nominal positions. $\Delta \theta$ is the displacement of the coil angle from its nominal value of $78^{\circ} . c_{x}$ and $c_{\theta}$ are scaling constants equal to $1 \mathrm{~m}$ and 1 radian, respectively. The target parameters $\mathbf{X}^{*}$ were generated using $p_{1}=0$ and $p_{2}=8 \times 10^{-3} ;$ i.e., no discrepancy in coil separation and a decrease of $0.46^{\circ}$ in $\theta_{\text {tilt }}$. The initial guess $\mathbf{p}_{0}$ was $(0,0)$ and the finite differencing interval for both components was $10^{-3}$.

The results of this test are shown in Fig. 8. Note that the contours of $\chi^{2}$ exhibit a shallow valley along a line consisting of a family of displacements that would result in similar Poincaré geometry. Nevertheless, the algorithm succeeded in moving the components of $\mathbf{p}$ to within one finite differencing interval of the target values on the fourth iteration.

Subsequent verifications tested the ability of the code to identify similar coil displacements when more coil parameters were free. In these tests, the IL coils were permitted to undergo rigid rotations and transformations; hence, each coil was allowed five degrees of freedom: three translational and two angular. The translational parameters, $x, y$, and $z$, are simply Cartesian displacements along the respective unit vectors indicated in Fig. 1. The angular parameters are illustrated in Fig. 9. As shown in the diagram, $a_{I L}$ and $b_{I L}$ are orthogonal projections of a unit vector representing the perturbed coil axis onto the plane of the unperturbed coil. The $a_{I L 1}$ and $b_{I L 1}$ components correspond to the directions $\hat{a}_{I L 1}$ and $\hat{b}_{I L 1}$ illustrated in Fig. 7. The polar displacement angle of the perturbed axis of the first IL coil can thus be computed as $\arcsin \left(\sqrt{{a_{I L 1}{ }^{2}+b_{I L 1}^{2}}^{2}}\right.$.

Fig. 10 shows the outcomes of two calculations that optimized the ten IL coil parameters as described above to a target $\mathbf{X}^{*}$ vector generated numerically from a chosen set $\mathbf{p}^{*}$ of displacements. In Fig. 10a, the only nonzero component of $\mathbf{p}^{*}$ was $z_{I L 1}$, which was set to $5 \mathrm{~mm}$, a simple translation of the first IL coil. In 


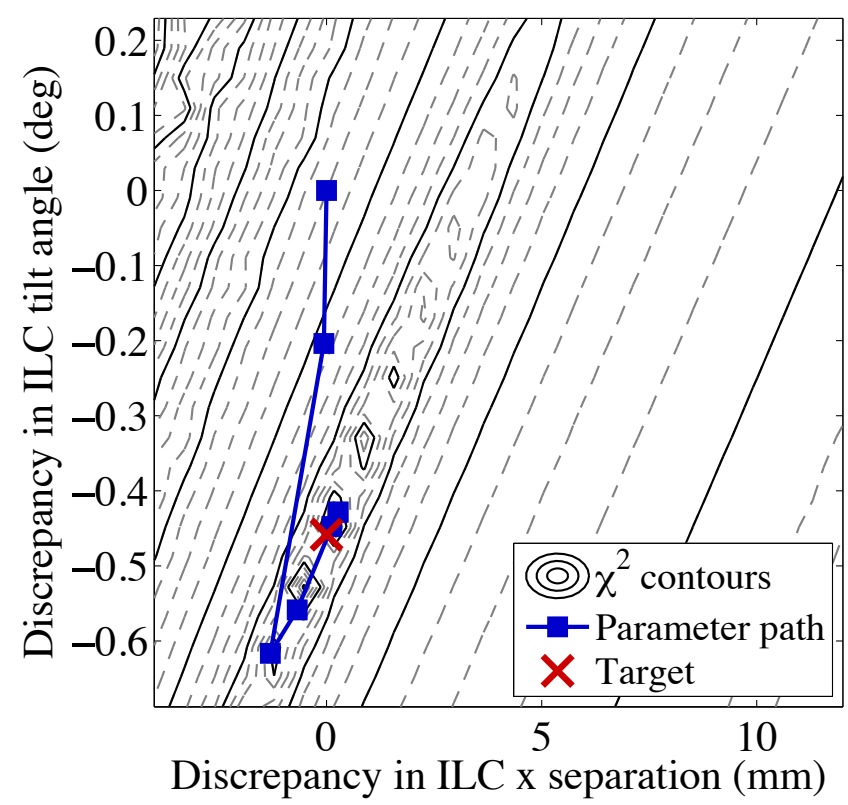

Figure 8. Contour plot of $\chi^{2}$ and the path taken by the optimizer in a verification test with two free parameters. The initial guess $\mathbf{p}_{0}$ is $(0,0)$ and the target parameters are denoted by the red $\times$. Contours are on a logarithmic scale with solid contours representing powers of 10 .

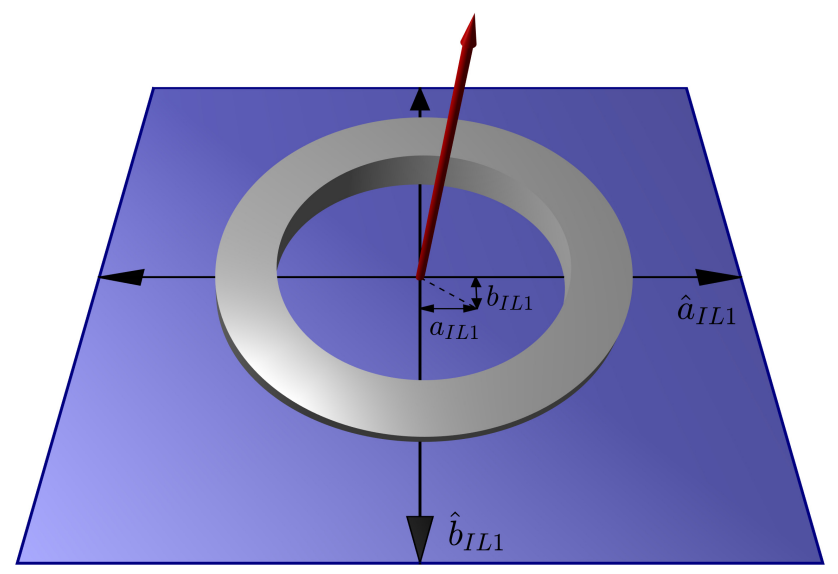

Figure 9. Schematic illustrating the definitions of the $a$ and $b$ components of the angular displacement parameters of the coils. In this image, the IL1 coil is used as an example. The blue surface represents the plane of the unperturbed coil, and the red arrow represents a unit vector along the axis of the perturbed coil. (a)

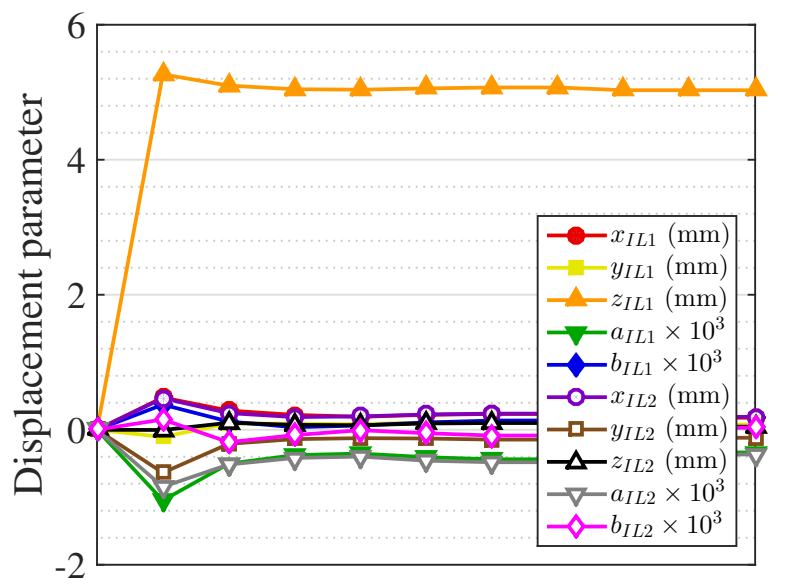

(b)

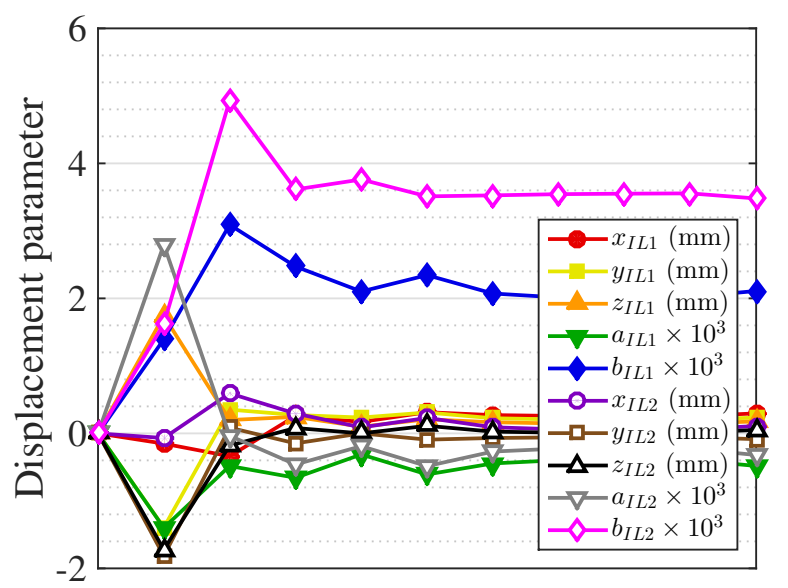

(c)

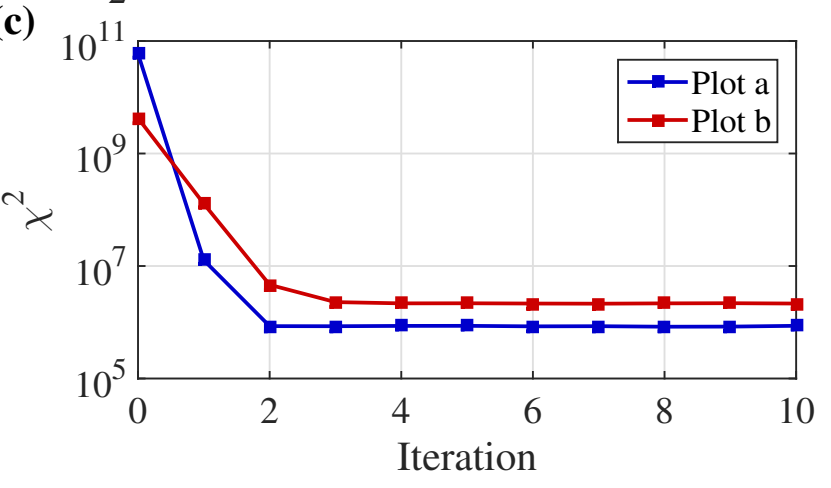

Figure 10. Results of optimizations in which the interlocked coils were free to move in 10 parameters to match Poincaré data generated from made-up coil perturbations. (a) Parameter evolution toward targets of $z_{I L 1}=5 \mathrm{~mm}$ and the rest of the parameters zero. (b) Parameter evolution toward targets of $b_{I L 1}=0.002, b_{I L 2}=0.004$, and the rest zero. (c) Descent of $\chi^{2}$ for both optimizations. 
Fig. 10b, there were two nonzero components of $\mathbf{p}^{*}$ : $b_{I L 1}=0.002$ and $b_{I L 2}=0.004 ;$ i.e., both coils were tilted toward their respective $\hat{b}$ vectors, but by different amounts.

Both optimizations descended by multiple orders of magnitude in $\chi^{2}$ in the first three steps (Fig. 10c), but afterward, $\chi^{2}$ flattened out: the optimizations did not converge any further toward $\mathbf{p}^{*}$. One possible explanation is that a relatively long finite differencing interval $\Delta \mathbf{p}$ was used to determine the Jacobian in each step: all translational $(x, y$, and $z)$ components used an interval of $1 \mathrm{~mm}$, and the angular components ( $a$ and $b$ ) used an interval of 0.001. All of the final values $p_{i}$ of the components are well within $\Delta p_{i}$ of the target values $p_{i}^{*}$ (Fig. 10a-b). Another contributing factor may be the numerical uncertainty of $\mathbf{F}$.

\subsection{Inferring coil displacements from experimental data}

After the verification tests, the algorithm was applied to experimental Poincaré data. The target geometric parameters $\mathbf{X}^{*}$ were determined from the cross-section data for the current-ratio 3.68 (Fig. B1). This dataset was chosen over datasets from other current-ratios for its abundance of fully-characterized flux surfaces and for its lack of magnetic islands, two attributes that facilitate accurate calculations of $\mathbf{X}^{*}$ (Sec. 6.1). The PF coils were held fixed with displacements determined by a best-fit to the photogrammetry data discussed in Sec. 3. All ten parameters associated with the IL coils were free. The initial guess $\mathbf{p}_{0}$ was the nominal IL coil configuration. As in the 10-parameter verifications conducted in Sec. 5.3, the finite differencing interval was $1 \mathrm{~mm}$ for all translational motion and 0.001 for all angular displacements. The set of displacements obtained in the final iteration will be referred to hereafter as $\mathbf{p}_{3.68}^{*}$.

The shifts in IL coil positions during the fifteen steps of the optimization are shown in Fig. 11a, with the accompanying descent of $\chi^{2}$ shown in Fig. 11b. The largest translational moves from the starting positions occurred in the negative $\hat{y}$ direction for both coils, averaging to $-22 \mathrm{~mm}$. The $\hat{z}$ displacement was nearly identical at around $6 \mathrm{~mm}$ for both coils, and the $\hat{x}$ displacement averaged to about $3 \mathrm{~mm}$ with a $3 \mathrm{~mm}$ "counter-" component (cf. the displacement classes in Fig. 6). It should be noted that, while the "co-" translational motion of the coils may indicate that the IL coils are indeed off of their nominal positions, it may also reflect misalignment of the fluorescent rod.

The largest angular shift was of the first IL coil, which tilted about $1^{\circ}$ away from its nominal axis along $\hat{a}_{I L 1}$, effectively decreasing $\theta_{\text {tilt }}$ to $77^{\circ}$. With the results of Fig. 6 in mind, this shift, combined with the $3 \mathrm{~mm}$ of counter-motion in the $\hat{x}$ direction, is
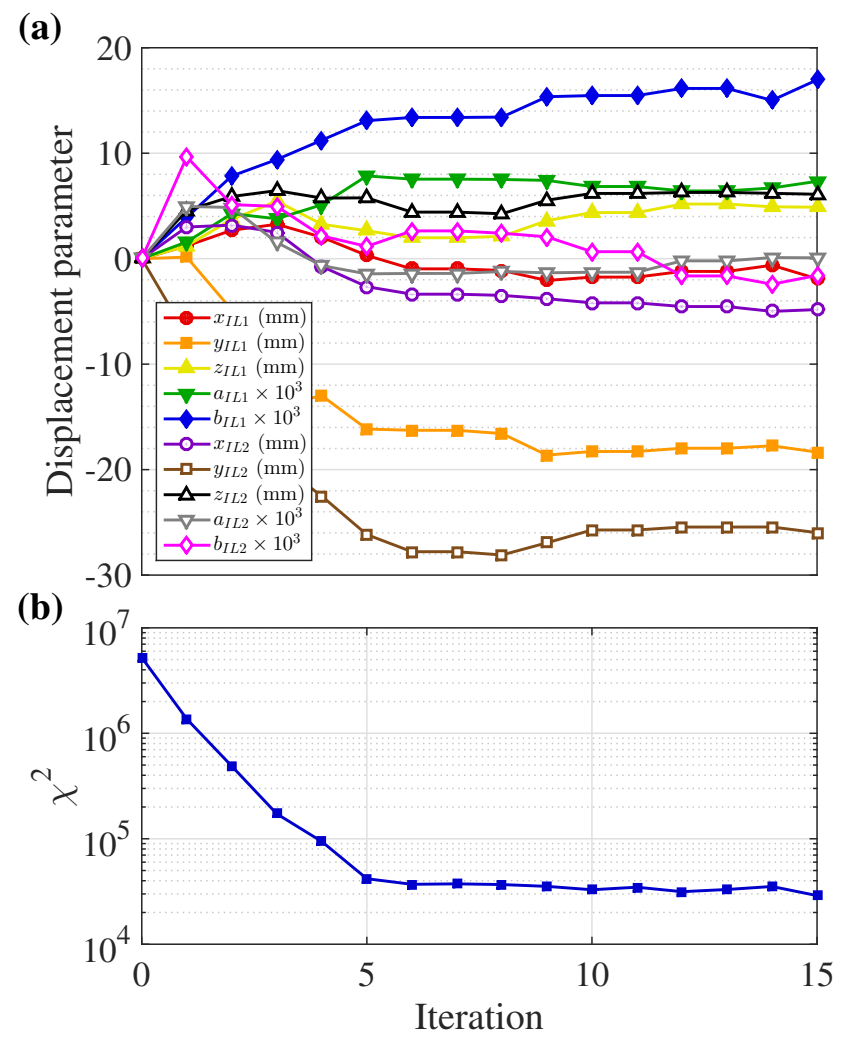

Figure 11. (a) Evolution of the displacement parameters of the IL coils during an optimization using experimental Poincaré data from the 3.68 current-ratio. (b) Descent of $\chi^{2}$ associated with the Poincaré data resulting from the displacements.

likely responsible for most of the downward offset in $t$ observed experimentally.

During the optimization, the value of $\chi^{2}$ (Fig. 11b) for the Poincaré data associated with the displacements decreased by a factor of more than 100. Most of this decrease occurred during the first five iterations, after which improvement was insignificant. The final value of $\chi^{2}$ is lower for this optimization than in the verifications (Fig. 10c) by about an order of magnitude; however, this is primarily due to the fact that the target parameters $\mathbf{X}^{*}$ determined here from the experimental data have greater uncertainty than those of the manufactured solutions.

The qualitative improvement in the numerically predicted Poincaré plots generated using $\mathbf{p}_{3.68}^{*}$ versus $\mathbf{p}_{0}$ is shown in Fig. 12. Fig. 12a, identical to Fig. 2a, refers to $I_{I L} / I_{P F}=3.68$ and compares the experimental data to a numerical prediction generated from $\mathbf{p}_{0}$ (i.e., coil displacements were neglected). Note, again, the disagreements in the vertical elongation on the outboard side, the concavity on the inboard side, and in the position of the magnetic axis. Most of the numerical cross-sections intersect multiple experimental ones. 
(a)

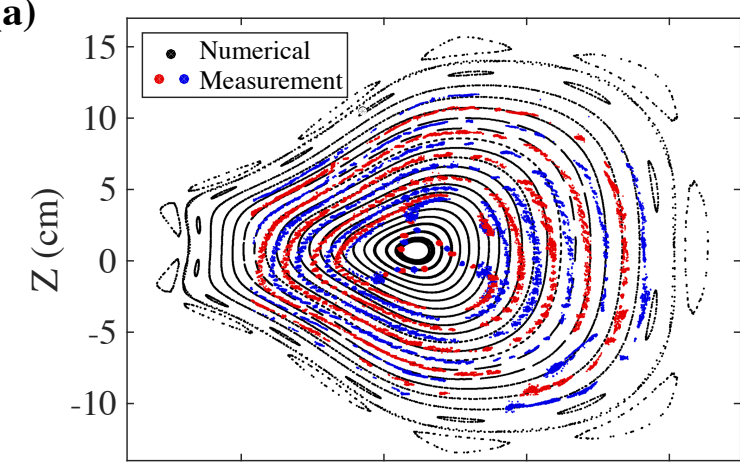

(b)

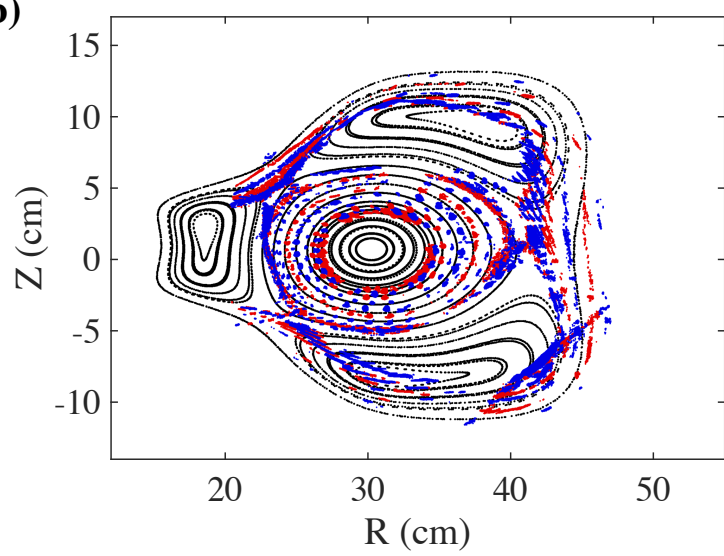

Figure 13. Effect of using, in calculations for (a) $I_{I L} / I_{P F}=$ 3.50 and (b) $I_{I L} / I_{P F}=3.18$, the coil displacements inferred for $I_{I L} / I_{P F}=3.68$ (i.e., $\left.\mathbf{p}_{3.68}^{*}\right)$. Measurements for the respective current ratios are shown for comparison.

Fig. $12 \mathrm{~b}$ shows the same experimental data overlaid on numerical data computed from $\mathbf{p}_{3.68}^{*}$. The incorporation of the displacements in the comparison has dramatically improved the qualitative agreement. The discrepancies in magnetic axis, as well as the shaping of the inboard and outboard sides, has essentially vanished, and there far fewer cases of numerical cross-sections intersecting multiple experimental ones.

Figs. 13a-b show comparisons at other currentratios $\left(I_{I L} / I_{P F}=3.50\right.$ for $13 \mathrm{a}$ and $I_{I L} / I_{P F}=3.18$ for $13 \mathrm{~b}$ ) in which the numerical Poincaré data were generated from $\mathbf{p}_{3.68}^{*}$. The agreement is not as good as in Fig. 12b, which is to be expected because the error vector $\mathbf{F}$ used in the optimization was based exclusively on the geometry of the $I_{I L} / I_{P F}=3.68$ cross-section. It is noteworthy, however, that for $I_{I L} / I_{P F}=3.18$ the optimization nonetheless predicts three islands near the edge of size and position comparable to what is observed. Note also the improvement in agreement for $I_{I L} / I_{P F}=3.18$ using $\mathbf{p}_{3.68}^{*}$ in Fig. $13 \mathrm{~b}$ over Fig. $2 \mathrm{~b}$ in which the numerical data were calculated from the nominal coil positions.

Finally, calculated values of $t$ at various current- ratios $I_{I L} / I_{P F}$ using $\mathbf{p}_{3.68}^{*}$ are shown in Fig. 14b. Note that, for $I_{I L} / I_{P F}<3.5$, the profiles of $t$ contain the value $1 / 3$, which is consistent with the observations described in Sec. 2.2. Recall that the $t$ profiles that had been predicted for the nominal coil positions (Fig. 3) failed to account for the presence of $t=1 / 3$ for $I_{I L} / I_{P F} \geq 3.18$ and that, furthermore, the PF coil displacements alone were insufficient to explain this disagreement (Fig. 5). Hence, the optimization of the IL coil displacements has resolved the discrepancy between the observations and predictions.

\section{Discussion}

\subsection{Optimizing for cross-sections with islands}

For the optimization conducted for the CNT IL coils in this paper, the target parameters in $\mathbf{X}^{*}$ came exclusively from a single current-ratio topology $\left(I_{I L} / I_{P F}=3.68\right)$ that had no significant magnetic islands. In principle, a cross-section containing magnetic islands should also be useable for an optimization if adequate experimental surface data are available, in the sense that many puncture points are distributed evenly around the cross-section of the surface. Such data were not available from CNT crosssections featuring prominent islands, largely as a result of two factors.

The first was that cross-sections containing the large $t=1 / 3$ island chain were cut off at the inboard side due to the finite extent of the fluorescent rod. On the other hand, had the rod extended further inboard, it would have collided with the IL coils during rotation. Thus, as seen in Fig. 13b, there are no complete surfaces recorded outside the island chain. This factor may be unique to CNT as it relates to the particular coil configuration.

The second factor, more likely to arise in other stellarators, is the shadowing of near-rational surfaces in the vicinity of island chains. This is a result of the electron beam striking the back side of the gun before reaching the fluorescent rod. This effect is visible in surfaces near the magnetic axis in Fig. 12a-b, in which only three dots appear, corresponding to the first three toroidal transits of the electron beam. In this case, the shadowing was not a major concern because only a small portion of the cross-section was affected. But in low-shear profiles with one or more rational surfaces at intermediate minor radii, large portions of the crosssection may be shadowed, thereby obscuring a large amount of geometric information and preventing the determination of accurate geometric parameters.

However, if the above factors are absent or limited in extent, we expect that cross-sections with island chains should be useable for optimization.

Poincaré data from field lines within a chain of 

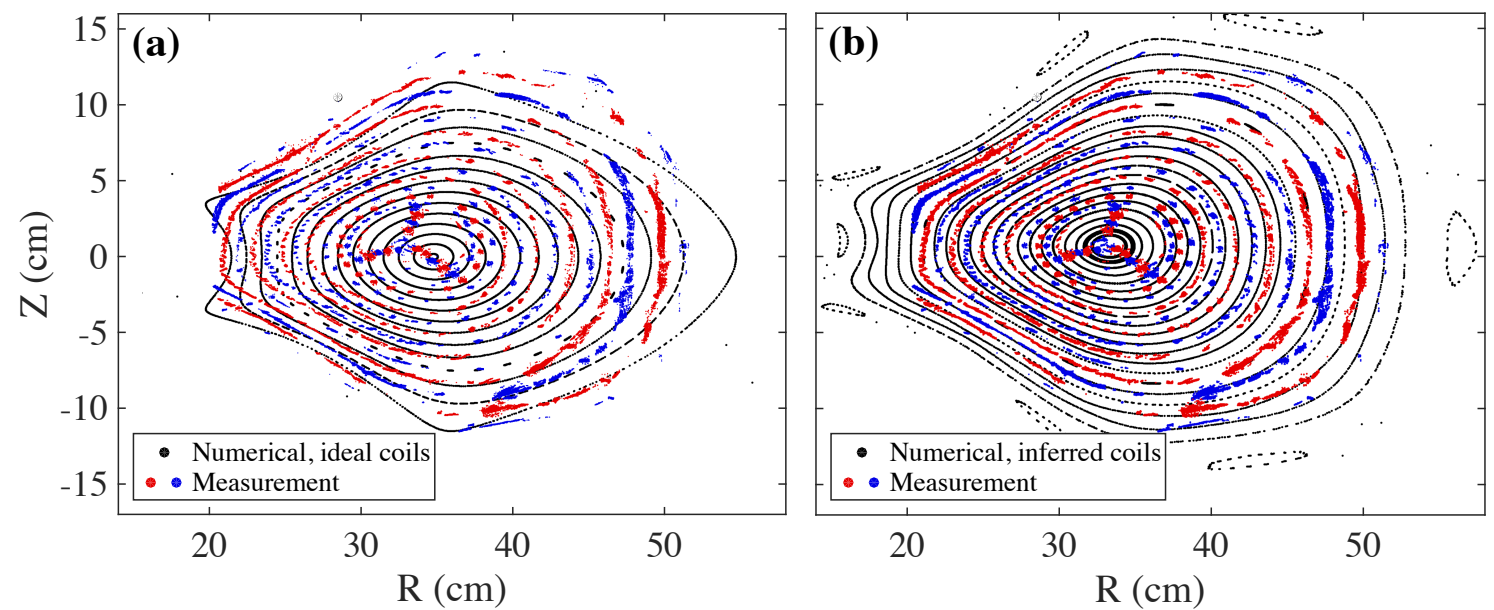

Figure 12. (a) Experimental data (red and blue dots) plotted alongside numerical data (black) for a field line trace that assumed no coil displacements, both at $I_{I L} / I_{P F}=3.68$, identical to what is plotted in Fig. 2a. (b) The same experimental data as in (a) plotted against numerical data for the coil displacements inferred from the optimization ( $\mathbf{p}_{3.68}^{*}$ ), again at $I_{I L} / I_{P F}=3.68$.

(a) Nominal coil positions

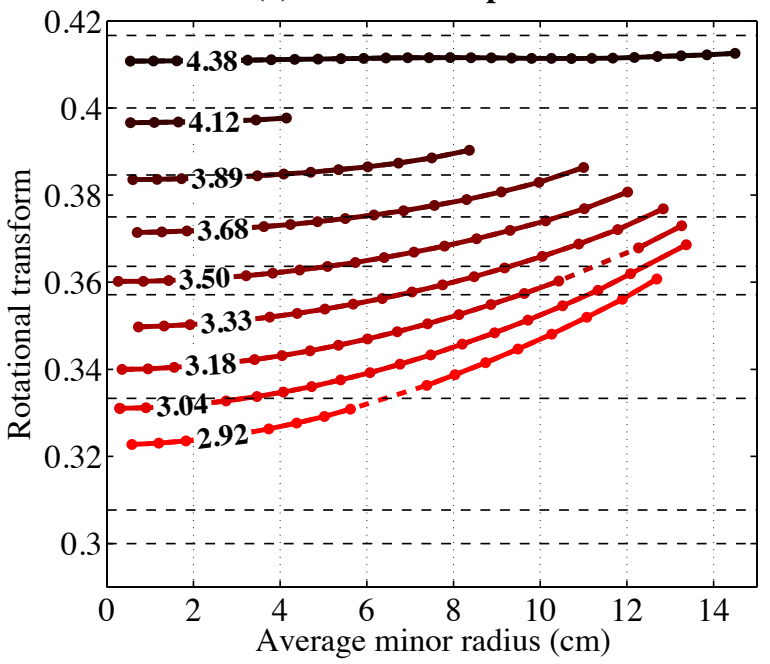

(b) Inferred coil positions

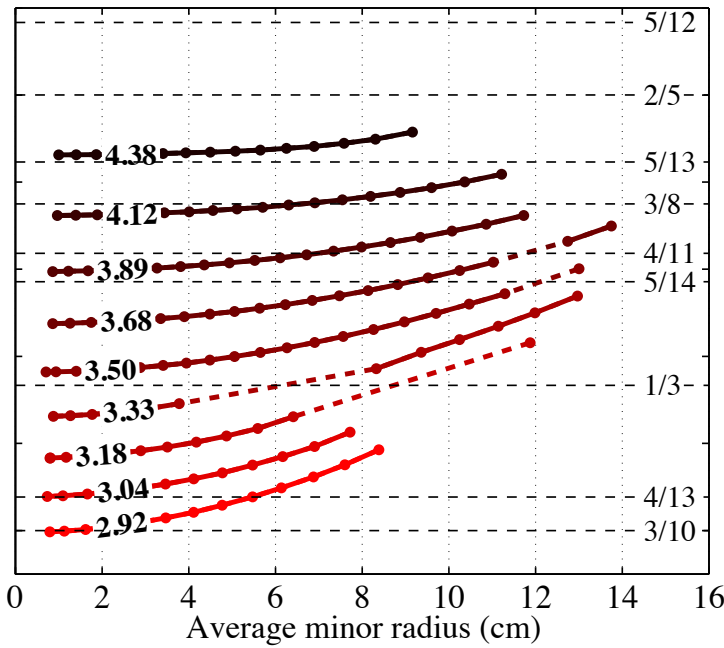

Figure 14. Comparison of $t$ profiles calculated for selected current-ratios in the nominal and optimized coil configurations. (a) Profiles for the nominal IL coil positions $\left(\mathbf{p}_{0}\right)$ and assuming no PF coil displacements, identical to what is plotted in Fig. 3 . (b) Profiles computed using the optimized IL coil configuration $\left(\mathbf{p}_{3.68}^{*}\right)$ and the measured PF coil displacements. Each dot represents a closed flux surface, so while the curves for $I_{I L} / I_{P F}=3.04$ and $2.92 \mathrm{in}$ plot (b) do not pass through $t=1 / 3$, they contain large three-island chains on their edges. Note that, in the optimized configuration (b), current-ratios $I_{I L} / I_{P F}$ less than 3.5 contain $t=$ $1 / 3$, consistent with observations.

large islands, identifiable by large empty intervals in the poloidal angle $\theta$, are ignored in the parametrization procedure (Appendix B.1). Thus, only the closed flux surfaces on either side of the island chain will determine $\mathbf{X}$, and, in turn, the Netwon steps $\delta \mathbf{p}$ in an optimization. Although the information about the magnetic geometry within the islands themselves is lost, the kinking of nearby closed flux surfaces adjacent to the island chain is retained. It is hypothesized that the closed flux surfaces with island-induced kinking should be sufficient for the optimization.

\subsection{Extension to more complex devices}

Although the vector $\mathbf{p}$ of coil parameters used for the optimization in this paper had only ten components, this vector can in principle be expanded to contain arbitrarily many parameters characterizing non-rigid displacements for multiple coils. One logical extension for CNT would be to allow for elliptical compression 
of the IL coils, which would add two more parameters for each coil (one for ellipticity and one for phase). More general deformations may be treated, for example, as Fourier series in which each additional $m$ number would require the addition of two parameters (coefficients) if the coil is assumed to stay planar. With the addition of even more parameters, the coil could be allowed to deform off of its nominal plane.

It follows that, for an arbitrary stellarator, the size of $\mathbf{p}$ would scale as the number of coils to be optimized times the number of degrees of freedom for rotation, translation and deformation afforded to each coil. Of course, as seen in the foregoing study, each coil need not be given the same number of degrees of freedom.

Assuming $\mathbf{X}$ consists of geometric data from only one current-ratio (which need not be the case), each column of the Jacobian can be computed with a single call to the field line tracer. Different columns, however, corresponding to perturbations to different components of $\mathbf{p}$, require separate field line traces. Since the field line traces are the most demanding part of the optimization procedure, the computation will scale linearly with the size of $\mathbf{p}$. It should be noted, however, that since the columns of the Jacobian may be determined in parallel with one another, the time need not scale linearly if multiple processors are available.

\section{Summary and future work}

In summary, significant vacuum field errors have been observed in the CNT stellarator with the coils configured to have $\theta_{\text {tilt }}=78^{\circ}$. Photogrammetric measurements of the PF coils showed misalignments, although those misalignments were not sufficient to fully explain the disagreements, in particular the observed offset in the $t$ profile. A numerical analysis of the influences of coil displacements on $t$ indicate that $t$ is most sensitive to displacements of the IL coils. This motivated the development of a numerical optimization method to calculate displacements for the IL coils that, in combination with the measured PF coil displacements, fit to the observed Poincaré crosssections. The application of this algorithm to CNT at $I_{I L} / I_{P F}=3.68$ led to a set of IL coil displacements that exhibited significantly improved quantitative and qualitative agreement with observations.

Future work will focus on improving the optimization algorithm to achieve even better fits to the observed data. These improvements will include (1) simultaneously optimizing to data from multiple currentratios using $\mathbf{F}$ vectors that include geometric parameters for multiple Poincaré cross-sections, (2) operating the field line tracer at greater numerical precision to reduce the uncertainty in numerically generated Fourier coefficients and allow for smaller finite difference inter- vals for the computation of the Jacobian, (3) expanding the $\mathbf{p}$ vector to include other sources of error including coil deformations, uncompensated coil leads, and displacements of the PF coils, and (4) investigating ways of generalizing the $\mathbf{X}$ vector to include information about Poincaré data inside islands, possibly analogous to generalizations of 3D toroidal equilibria found in codes like PIES [27], SIESTA [28], and SPEC [29]. Many of these improvements will be more demanding computationally, although much of the algorithm can be parallelized (in particular, the calculation of the covariance and Jacobian matrices).

While the misalignments in the CNT coils could in principle be corrected by, for example, repairing or replacing the support structures, this is not a high priority for the CNT program. One reason for this is that, in spite of the field errors, we still have access to configurations with good sets of nested, closed flux surfaces such as the one attained for $I_{I L} / I_{P F}=3.68$. In addition, the large island chains that the field errors have given rise to at lower current-ratios present an opportunity for research in island divertor physics.

\section{Acknowledgments}

The authors would like to thank S. Lazerson for assistance with the field line tracing code employed in this study, as well as R. Diaz-Pacheco and Y. Wei for their assistance with data collection. The authors would also like to acknowledge the financial support of the Department of Energy and the National Science Foundation of the United States, Grant No. NSF-PHY04-49813.

\section{References}

[1] Reimerdes H, Buttery R J, Garofalo A M, In Y, Haye R J L, Lanctot M J, Okabayashi M, Park J K, Schaffer M J, Strait E J and Volpe F A 2011 Fusion Science and Technology $\mathbf{5 9} 572-585$

[2] Hender T C, Wesley J C, Bialek J, Bondeson A, Boozer A H, Buttery R J, Garofalo A, Goodman T P, Granetz R S, Gribov Y, Gruber O, Gryaznevich M, Giruzzi G, Günther S, Hayashi N, Helander P, Hegna C C, Howell D F, Humphreys D A, Huysmans G T A, Hyatt A W, Isayama A, Jardin S C, Kawano Y, Kellman A, Kessel C, Koslowski H R, Haye R J L, Lazzaro E, Liu Y Q, Lukash V, Manickam J, Medvedev S, Mertens V, Mirnov S V, Nakamura Y, Navratil G, Okabayashi M, Ozeki T, Paccagnella R, Pautasso G, Porcelli F, Pustovitov V D, Riccardo V, Sato M, Sauter O, Schaffer M J, Shimada M, Sonato P, Strait E J, Sugihara M, Takechi M, Turnbull A D, Westerhof E, Whyte D G, Yoshino R, Zohm H, the ITPA MHD Disruption and Group M C T 2007 Nuclear Fusion 47 S128-S202

[3] Mynick H E 2006 Physics of Plasmas 13058102

[4] Xanthopoulos P, Mynick H E, Helander P, Turkin Y, Plunk G G, Jenko F, Görler T, Told D, Bird T and Proll J H E 2014 Physical Review Letters 113155001

[5] Sinclair R M, Hosea J C and Sheffield G V 1970 Applied Physics Letters $\mathbf{1 7} 92$ 
[6] Colchin R J, Anderson F S B, England A C, Gandy R F, Harris H H, Henderson M A, Hillis D L, Kindsfather R R, Lee D K, Million D L, Murakami M, Neilson G H, Saltmarsh M J and Simpson C M 1989 Review of Scientific Instruments 60 2680-2689

[7] Jaenicke R, Ascasibar E, Grigull P, Lakicevic I, Weller A, Zippe M, Hailer H and Schwörer K 1993 Nucl. Fusion 33 687-704

[8] Pedersen T S, Kremer J P, Lefrancois R G, Marksteiner Q, Sarasola X and Ahmad N 2006 Phys. Plasmas 13012502

[9] Colchin R J, Harris J H, Anderson F S B, England A C, Gandy R F, Hanson J D, Henderson M A, Hillis D L, Jernigan T C, Lee D K, Lynch V E, Murakami M, Neilson G H, Rome J A, Saltmarsh M J and Simpson C M 1989 Correction of field errors in the atf torsatron Proceedings of the 16th European Conference on Controlled Fusion and Plasma Physics, Venice (Europhysics Conference Abstracts vol 13B) (IAEA) pp 615-618

[10] Otte M, Lingertat J and Wagner F 2003 Magnetic flux surface measurements with vertical field and compensation coils at the wega stellarator Proceedings of the 14th International Stellarator Workshop, Greifswald

[11] Sakakibara S, Narushima Y, Okamoto M, Watanabe K Y, Suzuki Y, Ohdachi S, Ida K, Yoshinuma M, Tanaka K, Tokuzawa T, Narihara K, Yamada I, Yamada H and the LHD Experiment Group 2013 Nuclear Fusion 53043010

[12] Hirsch M, Baldzuhn J, Beidler C, Brakel R, Burhenn R, Dinklage A, Ehmler H, Endler M, Erckmann V, Feng Y, Geiger J, Giannone L, Geiger G, Grigull P, Hartfuss H J, Hartmann D, Jaenicke R, König R, Laqua H P, Maassberg H, McCormick K, Sardei F, Speth E, Stroth U, Wagner F, Weller A, Werner A, Wobig H, Zoletnik S and the W7-AS Team 2008 Plasma Physics and Controlled Fusion $\mathbf{5 0} 053001$

[13] Rummel T, Risse K, Kisslinger J, Köppen M, Füllenback F, Neilson H, Brown T and Ramakrishnan S 2012 IEEE Transactions on Applied Superconductivity $22420 \overline{1704}$

[14] Kisslinger J and Andreeva T 2005 Fusion Engineering and Design 74 623-626

[15] Landreman M and Boozer A H 2016 Phys. Plasmas 23 032506

[16] Pedersen T S, Boozer A H, Kremer J P, Lefrancois R G, Reiersen W T, Dahlgren F D and Pomphrey N 2004 Fusion Sci. Technol. 46200

[17] Imagawa S, Masuzaki S, Yanagi N, Yamaguichi S, Satow T, Yamamoto J, Motojima O and the LHD group 1998 Fusion Engineering and Design 41253

[18] Wanner M and the W7-X team 2000 Plasma Physics and Controlled Fusion 421179

[19] Lazerson S, Otte M, Bozhnekov S, Biedermann C, Pedersen T S and the W7-X team 2016 Nuclear Fusion (submitted)

[20] Brenner P W, Pedersen T S, Berkery J W, Marksteiner Q R and Hahn M S 2008 IEEE Transactions on Plasma Science 361108

[21] Kremer J P 2006 The creation and first studies of electron plasmas in the Columbia Non-neutral Torus Ph.D. thesis Columbia University New York, NY 10027

[22] Lao L L, John H S, Stambaugh R D, Kellman A G and Pfeiffer W 1985 Nuclear Fusion 251611

[23] Hanson J D, Hirshman S P, Knowlton S F, Lao L L, Lazarus E A and Shields J M 2009 Nuclear Fusion 49075031

[24] Press W H, Teukolsky S A, Vetterling W T and Flannery B P 1992 Numerical Recipes in C 2nd ed (Cambridge University Press)

[25] Jones C S and Finn J M 2006 Nuclear Fusion 46335

[26] James G, Witten D, Hastie T and Tibshirani R 2013 An Introduction to Statistical Learning with Applications in $\mathrm{R}$ (Springer)

[27] Rieman A and Greenside H 1986 Computer Physics Communications $\mathbf{4 3} 157$
[28] Hirshman S P, Sanchez R and Cook C R 2011 Physics of Plasmas 18062504

[29] Hudson S R, Dewar R L, Dennis G, Hole M J, McGann M, von Nessi G and Lazerson S 2012 Physics of Plasmas 19 112502

\section{Appendix A. Parametrization of Poincaré cross-sections}

The level of agreement between two sets of Poincaré data is determined by fitting the nested flux surface cross-sections to a discrete set of geometric parameters $\mathbf{X}$. The components of $\mathbf{X}$ are the coefficients of a linear combination of orthogonal functions consisting of a Fourier series in the poloidal angle $\theta$ and a polynomial series in the normalized minor radius $\rho$.

For a flux surface characterized by some particular $\rho$, the $R$ and $Z$ coordinates of the point with poloidal angle $\theta$ are expressed as

$$
\begin{aligned}
R(\rho, \theta)= & R_{0}(\rho)+\sum_{m=1}^{M} R_{c m}(\rho) \cos (m \theta) \\
& +\sum_{m=1}^{M} R_{s m}(\rho) \sin (m \theta) \\
Z(\rho, \theta)= & Z_{0}(\rho)+\sum_{m=1}^{M} Z_{c m}(\rho) \cos (m \theta) \\
& +\sum_{m=1}^{M} Z_{s m}(\rho) \sin (m \theta)
\end{aligned}
$$

As indicated in the above representation, the coefficients of each Fourier mode are themselves functions of $\rho$. This dependence is represented as a linear combination of polynomials $P_{s}(\rho)$; for example:

$R_{c 1}(\rho)=R_{c 10} P_{0}(\rho)+R_{c 11} P_{1}(\rho)+\ldots+R_{c 1 S} P_{S}(\rho)(\mathrm{A} .3)$

The polynomials $P_{s}(\rho)$ are chosen to be orthonormal in the inner product defined by

$$
\left\langle P_{i}, P_{j}\right\rangle=\int_{0}^{1} P_{i}(\rho) P_{j}(\rho) d \rho=\delta_{i j},
$$

and the first few polynomials in this set are listed in Table A1.

The vector $\mathbf{X}$ of geometric parameters, then, is just a list of these coefficients:

$$
\begin{aligned}
\mathbf{X}=\{ & R_{00}, \ldots, R_{0 S}, R_{c 10}, \ldots, R_{c 1 S}, \ldots, R_{c M S}, \\
& \left.R_{s 01}, R_{s M S}, Z_{00}, \ldots, Z_{s M S}\right\}
\end{aligned}
$$

To ensure that the set of coefficients is unique for a particular set of Poincaré data, it is necessary to use a precise definition for $\rho$ and $\theta$. In this work, $\rho$ for a particular cross-section is defined as $\sqrt{A / A_{\max }}$, where $A$ is the enclosed area and $A_{\max }$ is a reference 


\begin{tabular}{|l|l|}
\hline$s$ & $P_{s}(\rho)$ \\
\hline 0 & 1 \\
1 & $\sqrt{3}(2 \rho-1)$ \\
2 & $\sqrt{5}\left(6 \rho^{2}-6 \rho+1\right)$ \\
3 & $\sqrt{7}\left(20 \rho^{3}-30 \rho^{2}+12 \rho-1\right)$ \\
4 & $3\left(70 \rho^{4}-140 \rho^{3}+90 \rho^{2}-20 \rho+1\right)$ \\
\hline
\end{tabular}

Table A1. The first few polynomials $P_{s}(\rho)$ as discussed in the text.

area not to exceed the area enclosed by the last closed flux surface. In this way, $\rho=0$ at the magnetic axis and $\rho=1$ on the edge of the region of the Poincaré cross-section to be parametrized. The poloidal angle $\theta$ is defined to advance in direct proportion to the arc length along a flux surface cross-section and is set to zero on the outboard side where $Z$ is equal to $Z(\rho=0)$.

\section{Appendix B. Determination of the $\mathrm{X}$ vector for Poincaré data}

An example of a fit of coefficients to experimental data is shown in Fig. B1. As discussed in Section 2 , the experimental data were separated according to flux surface. For each flux surface, a curve was fit iteratively to the Poincaré points in such a way as to minimize the disagreement with the data points. An example of a set of fit curves is shown in Fig. B1a. When the fitting iterations are complete, the curve was parametrized in $\theta$ as described in Appendix A and the $i^{t h}$ measured surface was assigned a $\rho_{i}$ value based on the enclosed area. Each fit curve $\left(R\left(\rho_{i}, \theta\right), Z\left(\rho_{i}, \theta\right)\right)$ was then projected onto a Fourier series, yielding one set of coefficients $\left\{R_{c m}\left(\rho_{i}\right), R_{s m}\left(\rho_{i}\right), Z_{c m}\left(\rho_{i}\right), Z_{c m}\left(\rho_{i}\right)\right\}_{m=0}^{M}$ for each value $\rho_{i}$. Sets of corresponding Fourier coefficients (e.g., $\left.\left\{R_{c 1}\left(\rho_{i}\right)\right\}_{i}\right)$ were then fit by linear least-squares onto polynomials in $\rho$ of degree $S$, from which the polynomial coefficients as shown in Eq. A.3 were obtained. The calculations described in this paper used $M=14$ and $S=7$.

Because experimental data points are often limited near the magnetic axis, certain constraints are enforced in the least-squares fitting in order to avoid spurious oscillations in the $\rho$ polynomials. In particular, all polynomials for Fourier coefficients of $m \geq 1$ are constrained to be zero at the axis. In addition, all coefficients of $m \geq 2$ are constrained to have a first derivative of zero at the axis. Furthermore, coefficients with $m=0$ are only expanded to second order in $\rho$ (i.e., requiring $s \leq 2$ ).

Once coefficients are calculated for a set of (a)
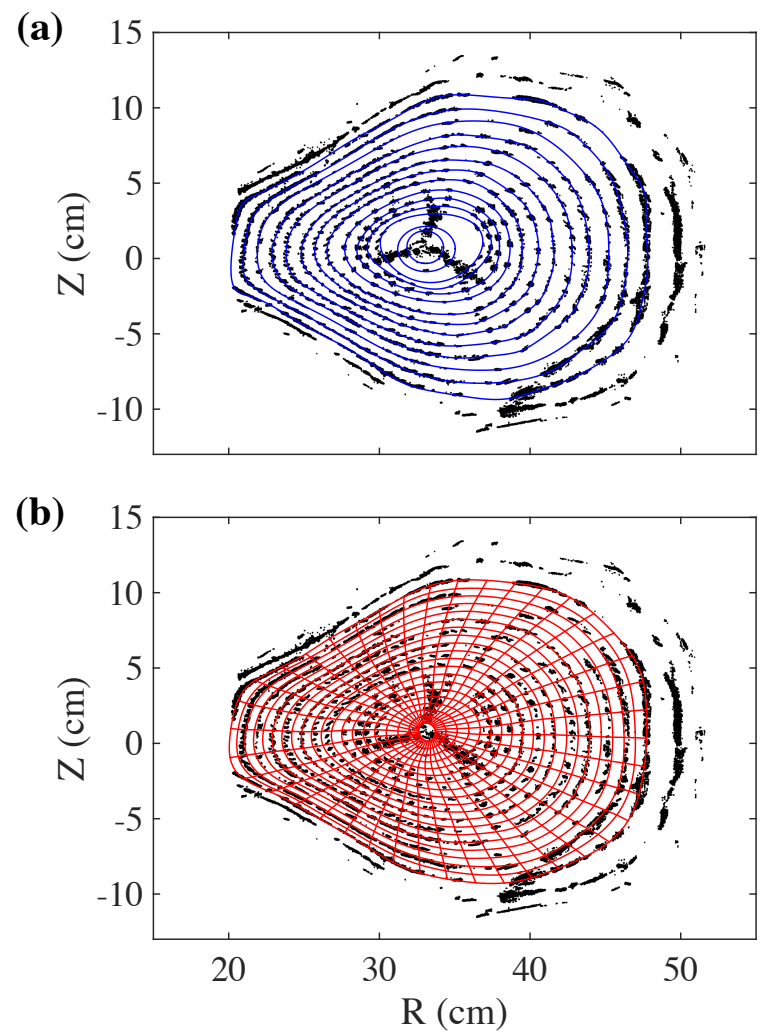

Figure B1. (a) Experimental Poincaré data obtained for the current-ratio 3.68 (black dots) and fit curves to selected flux surfaces (blue lines). (b) The same experimental data overlaid with level curves from the fitted geometric parameters in red. Radially extending red lines are curves of constant $\theta$; closed red loops are curves of constant $\rho$.

Poincaré data, they are arranged into a vector $\mathbf{X}$ containing $(S+1)(4 M+2)$ elements. Because of the constraints described in the above paragraph, $2(S-$ 2) $+8 M-4$ of those components are redundant, and are therefore removed for subsequent analysis.

Ideally, the coefficients $\mathbf{X}$ should be unique for a given set of Poincaré data. However, in practice the coefficients will vary slightly depending on which Poincaré points are used for this fit. Specifically, for experimental data, this depends on which surfaces are measured and which of the subsequent pixels are used for the analysis. For numerical data, the dependence is on the locations where the field line traces are initialized. Hence, each component of $\mathbf{X}$ will have an associated uncertainty that may be correlated with that of other components. Sec. 5.1 and 5.2 describe how these correlations are accounted for in the optimization.

\section{Appendix B.1. Treatment of magnetic islands}

If a chain of large islands exists within a crosssection, it will be identified during the curve-fitting 
process by poloidal gaps in the Poincaré data. Since the parametrization described in Appendix A only permits closed flux surfaces, the islands are simply ignored during in the fits of Fourier coefficients to the polynomials $P_{s}(\rho)$ decribed above. Since some Fourier coeffients may change abruptly from the axisfacing side of the island to the edge-facing side, it may be necessary to incorporate higher-order polynomials $P_{s}(\rho)$ in the fits, leading to more components in the $\mathbf{X}$ vector.

If one were to plot the curves of constant $\rho$ specified from the geometric parameters $\mathbf{X}$ (as in Fig. B1b), one would observe a continuous deformation from the shape of the last closed flux surface on the axis-facing side of the island chain to the first closed surface on the edge-facing side. These deformed curves in the island region clearly do not reflect the actual island geometry and are simply a result of interpolation between the data for the core-facing side and the edgefacing side. In other words, the laminar flux surfaces occurring in place of the islands reflect the fact that the $\mathbf{X}$ vector contains information only about the flux surfaces on either side of the island chain. 